\title{
Electron Positron Capture Rates and the Steady State Equilibrium Condition for Electron-Positron Plasma with Nucleons
}

\author{
Ye-Fei Yuan* \\ Center for Astrophysics, University of Science and \\ Technology of China, Hefei, Anhui 230026, P.R. China
}

\begin{abstract}
The reaction rates of the beta processes for all particles at arbitrary degeneracy are derived, and an analytic steady state equilibrium condition $\mu_{n}=\mu_{p}+2 \mu_{e}$ which results from the equality of electron and positron capture rates in the hot electron-positron plasma with nucleons is also found, if the matter is transparent to neutrinos. This simple analytic formula is valid only if electrons are nondegenerate or mildly degenerate, which is generally satisfied in the hot electron-positron plasma. Therefore, it can be used to efficiently determine the steady state of the hot matter with plenty of positrons. Based on this analytic condition, given the baryon number density and the temperature, if the nucleons are nondegenerate, only one algebraic equation for determining the electron fraction is obtained, which shows the great advantage of the analytic equilibrium condition.
\end{abstract}

PACS numbers: $13.15 .+\mathrm{g}, 26.30 .+\mathrm{k}$

*Electronic address: yfyuan@ustc.edu.cn 


\section{INTRODUCTION}

Gamma Ray Bursts (GRBs) 1] and core collapse supernovae(SNe) 2] are two of the most violent events in our universe. Ironically, their explosion mechanisms are still mysterious. Recently, the central engine of GRBs is believed to be related to the hyperaccretion of a stellar-mass black hole at extremely high rates from $\sim 0.01$ to $10 M_{\odot} \mathrm{S}^{-1}$ [3, 4, 5]. In such an accretion disk, matter is so dense that photons are trapped. The possible channel for energy release is either neutrino emission which is mainly from the electron-positron $\left(e^{ \pm}\right)$capture on nucleons and $e^{ \pm}$annihilation, or outflows from the disk. Whatever a successful central engine is, it ejects a hot fireball which consists of the radiation field ( $e^{ \pm} /$photons $)$and baryons. The ratio of neutrons to protons, or equivalently, the electron fraction, is crucial to the observed radiation from GRBs [7, 8], its dynamic evolution [5, 9] and the nucleosynthesis in the disk

or fireball [4, 5]. For instance, the inelastic collisions between neutrons and protons produce observable multi-GeV neutrino emission [1, 10]. In addition, the two component fluid of neutrons and protons significantly changes the fireball interaction with an external medium which is supposed to produce the observed electromagnetic radiation from GRBs and their afterglows [12]; and the electron fraction $Y_{e}$ strongly affects the equation of state of the hyperaccretion disk and the neutrino emissions from it.

Roughly speaking, SNe are powered by the iron core collapse of their progenitors. Most numerical simulations have shown not only the failure of the prompt shock, but the failure of its revival by the delayed neutrino emission from the protoneutron star (PNS). The result (explosion or not) sensitively depends on the input microphysics, such as the electron capture, the neutrino emission, neutrino-matter interactions, the equation of state, rotation, magnetic field, general relativity effects, and so on (see ref. [2], and references therein). Without a doubt, weak interactions, especially $e^{ \pm}$captures and neutron decay, play a key role in both GRBs and SNe. During the accretion or collapse, these processes exhaust electrons, thus decrease the degenerate pressure of electrons. Meanwhile, they produce neutrinos which carry the binding energy away to power the explosions. Therefore, electron capture is crucial to the formation of the bounce shock of SNe, and the resulting neutrino spectra strongly influence the neutrino-matter interactions which are energy dependent and are essential for collapsing simulations [13].

The existence of a hot state with nucleons is the common characteristics of both GRBs and 
SNe, as well as the PNS, the bounce shock and the early universe [14]. In these systems, the beta reactions are the most important physical processes [15]. If the system is transparent to neutrinos, the steady state is achieved via the following beta reactions [16],

$$
\begin{aligned}
e^{-}+p & \rightarrow n+\nu_{e}, \\
e^{+}+n & \rightarrow p+\bar{\nu}_{e}, \\
n & \rightarrow p+e^{-}+\bar{\nu}_{e} .
\end{aligned}
$$

These beta reaction rates are calculated in the previous studies, usually under one of three approximations: the nondegenerate approximation [15], the degenerate approximation [11, 20], and the elastic approximation in which there is no energy transfer to nucleons [21]. In this paper, applying the structure function formalism developed by Reddy et al. [22] (see also [23]), I derive the reaction rates of the beta processes in the dense subnuclear matter for all particles at arbitrary degeneracy. In addition, I find an analytic expression for determining the kinetic equilibrium between electron capture and positron capture, which is efficient to determine the steady state of the hot matter with plenty of positrons. If the neutrinos are partially trapped, neutrino transport should be considered carefully, which is out of the scope of this work [17].

\section{REACTION RATES.}

The lowest order Feynman diagrams for reactions (11)-(3) are shown in Fig. 11. Because in our consideration the energies of leptons are less than a few hundred $\mathrm{MeV}$ which is greatly less than the rest mass of $W^{ \pm}$mesons, thus the interaction Lagrangian from WeinbergSalam theory is reduced to the original Fermi's current-current interaction form. From Fermi's golden rule, the reaction rates of the processes (11)-(3) read (we set $\hbar=c=k_{\mathrm{B}}=1$ )

$$
\lambda=2 \int \prod_{i=1}^{4}\left[\frac{d^{3} \overrightarrow{p_{i}}}{(2 \pi)^{3}}\right](2 \pi)^{4} \delta^{(4)}\left(P_{i}-P_{f}\right)|M|^{2} \mathcal{F},
$$

where $P_{i}=\left(E_{i}, \vec{p}_{i}\right)$ denotes the four-momentum of particle $i\left(i=\nu_{e} / \bar{\nu}_{e}, e^{-} / e^{+}, n, p\right)$, $p_{i}=\left|\vec{p}_{i}\right|$, and $P_{i}$ and $P_{f}$ are the total initial and final momentum, respectively. $\mathcal{F}$ denotes the final-states blocking factor. In reaction $(1)-(3), \mathcal{F}=f_{e} f_{p}\left(1-f_{n}\right), \mathcal{F}=f_{e^{+}} f_{n}\left(1-f_{p}\right)$ , $\mathcal{F}=f_{n}\left(1-f_{p}\right)\left(1-f_{e}\right)$ respectively, where $f_{i}$ is the Fermi-Dirac function of particle $i$. In this paper, we just consider the case that the emitted neutrinos can escape freely 


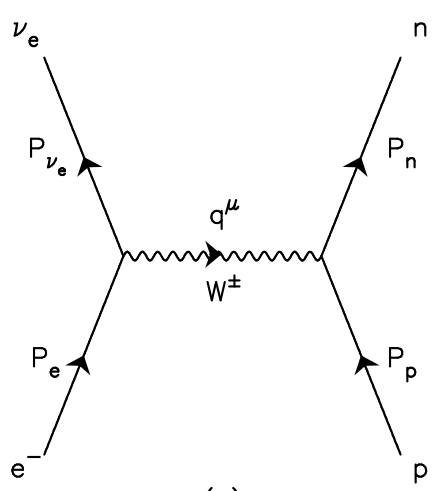

(a)

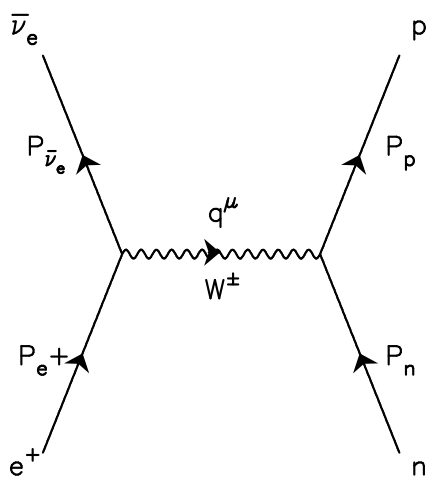

(b)

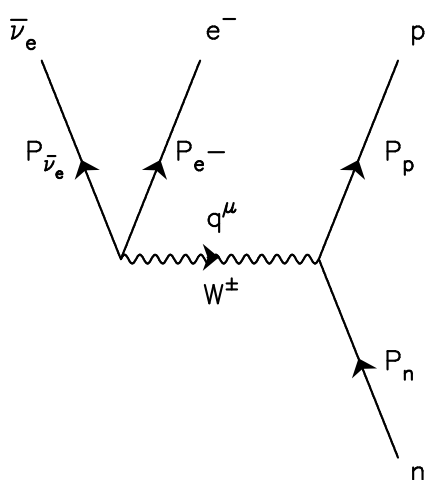

(c)

FIG. 1: The lowest order Feynman diagrams for $\beta$ processes. (a) Electron capture by proton: $e^{-}+p \rightarrow n+\nu_{e}$, the energy conservation requires $E_{e}=q_{0}+E_{\nu}$. (b) Positron capture by neutron: $e^{+}+n \rightarrow p+\overline{\nu_{e}}$, the energy conservation requires $E_{e^{+}}=q_{0}+E_{\bar{\nu}}$. (c) Neutron decay: $n \rightarrow p+e^{-}+\overline{\nu_{e}}$, the energy conservation requires $E_{e}=-q_{0}-E_{\bar{\nu}}$.

from the system. $|M|^{2}$ in the above equation is the averaged transition rate which depends on the initial and final states of all participating particles. Screening corrections to the electron capture rates in dense astrophysical environments have been investigated by many authors [18]. In the hot and dense $n p e^{ \pm}$gas, the Debye radius $r_{\mathrm{D}}$ is about $3 \mathrm{fm}\left(T / 10^{10} \mathrm{~K}\right)^{1 / 2}\left(n_{\mathrm{B}} / 0.01 \mathrm{fm}^{-3}\right)^{-1 / 2}\left(Y_{e} / 0.5\right)^{-1 / 2}$, where $n_{\mathrm{B}}=n_{n}\left(\mu_{n}, T\right)+n_{p}\left(\mu_{p}, T\right)$ is the baryon number density. Thus in our calculations, Coulomb waves are approximately replaced with plane waves for charged particles. As argued in Reddy et al. 22], for nonrelativistic noninteracting baryons $\left(n_{\mathrm{B}}<5 n_{\text {nuc }}\right)$, where $n_{\text {nuc }}=0.16 \mathrm{fm}^{-3}$ is the empirical nuclear equilibrium density, the transition rate averaged over the initial spins becomes a constant, i.e. $|M|^{2}=G_{\mathrm{F}}^{2} \cos ^{2} \theta_{\mathrm{C}}\left(1+3 g_{\mathrm{A}}^{2}\right)$, here $G_{\mathrm{F}} \simeq 1.436 \times 10^{-49}$ erg $\mathrm{cm}^{3}$ is the Fermi weak interaction constant, $\theta_{\mathrm{C}}\left(\sin \theta_{\mathrm{C}}=0.231\right)$ is the Cabibbo angle, and $g_{\mathrm{A}}=1.26$ is the axial-vector coupling constant. For the case of relativistic interacting baryons, the transition rate is expressed in terms of the target particle retarded polarization tensor in the relativistic mean field theory for interacting baryons, the resulting expression is so complicated (for more detail, see [19, 22]). In this paper, the beta reactions at subnuclear density are considered, therefore, Taking $|M|^{2}$ to be constant is a good approximation.

Using the structure function formalism developed by Reddy et al. 22], the above integrations can be simplified into only three dimensional ones, therefore, the rate of the $e^{ \pm}$-captures 
$\lambda_{e^{-} p}, \lambda_{e^{+} n}$, and the rate of neutron decay $\lambda_{n}$ are given by

$$
\begin{aligned}
& \lambda_{e^{-} p}=\frac{1}{8 \pi^{4}}|M|^{2} \int_{0}^{\infty} d E_{\nu} \int_{m_{e}-E_{\nu}}^{\infty} d q_{0} \\
& \times \int_{\left|p_{e}-p_{\nu}\right|}^{\left|p_{e}+p_{\nu}\right|} d q E_{\nu} E_{e} f_{e} S_{p \rightarrow n}\left(q_{0}, q\right) q, \\
& \lambda_{e^{+} n}=\frac{1}{8 \pi^{4}}|M|^{2} \int_{0}^{\infty} d E_{\bar{\nu}} \int_{m_{e}-E_{\bar{\nu}}}^{\infty} d q_{0} \\
& \times \int_{\left|p_{e^{+}}-p_{\bar{\nu}}\right|}^{\mid p_{e^{+}+p_{\bar{\nu}} \mid}} d q E_{\bar{\nu}} E_{e^{+}} f_{e^{+}} S_{n \rightarrow p}\left(q_{0}, q\right) q, \\
& \lambda_{n}=\frac{1}{8 \pi^{4}}|M|^{2} \int_{0}^{\infty} d E_{\bar{\nu}} \int_{-\infty}^{-\left(m_{e}+E_{\bar{\nu}}\right)} d q_{0} \\
& \times \int_{\left|p_{e}-p_{\bar{\nu}}\right|}^{\left|p_{e}+p_{\bar{\nu}}\right|} d q E_{\bar{\nu}} E_{e}\left(1-f_{e}\right) S_{n \rightarrow p}\left(q_{0}, q\right) q,
\end{aligned}
$$

where $S_{i \rightarrow j}\left(q_{0}, q\right)$ is the so-called dynamic form factor or structure function which characterizes the isospin response of the system [22], and $q_{0}=E_{f}-E_{i}, q=|\vec{q}|=\left|\overrightarrow{p_{f}}-\overrightarrow{p_{i}}\right|$ denote the momentum and energy transfer.

The expression $S_{i \rightarrow j}\left(q_{0}, q\right)$ is given by

$$
S_{i \rightarrow j}\left(q_{0}, q\right)=\frac{m_{i} m_{f} T}{\pi q} \frac{z+\xi_{-}}{1-\exp (-z)},
$$

where

$$
\begin{aligned}
z & =\frac{q_{0}+\mu_{i}-\mu_{j}}{T} \\
\xi_{-} & =\ln \left[\frac{1+\exp \left(\left(E_{-}^{i}-\mu_{i}\right) / T\right)}{1+\exp \left(E_{-}^{i}+q_{0}-\mu_{j}\right)}\right], \\
E_{-}^{i} & =m_{i}+\frac{m_{j}^{2}\left(q_{0}+m_{i}-m_{j}-q^{2} / 2 m_{j}\right)^{2}}{2 m_{i} q^{2}},
\end{aligned}
$$

where $\mu_{i}$ and $m_{i}$ are the chemical potential and the mass of baryons. In Eqs. (15)-(7), $E_{e}=q_{0}+E_{\nu}, E_{e^{+}}=q_{0}+E_{\bar{\nu}}$, and $E_{e}=-q_{0}-E_{\bar{\nu}}$ (see Fig. 1). Equations (15)-(7) are valid for nonrelativistic and noninteracting baryons [22]. Below the nuclear density, this is a good approximation. It should be emphasized that Eqs. (8)-(11) differ from the corresponding equations in Reddy et al. [22] in which the mass difference between nucleons is neglected. However, in order to investigate the rate of neutron decay, it is necessary to keep the mass difference in Eqs. (8)-(11).

Analogous to the analysis in Reddy et al. 22], it is easy to obtain the previous results in the nondegenerate and degenerate limits of baryons. For instance, the results in the 
nondegenerate limit corresponding to Eqs. (15)-(17) are shown below,

$$
\begin{aligned}
\lambda_{e^{-} p} \simeq & \frac{1}{2 \pi^{3}}|M|^{2} n_{p} \int_{Q}^{\infty} d E_{e} E_{e} p_{e}\left(E_{e}-Q\right)^{2} f_{e} \\
\lambda_{e^{+} n} \simeq & \frac{1}{2 \pi^{3}}|M|^{2} n_{n} \int_{m_{e}}^{\infty} d E_{e} E_{e} p_{e}\left(E_{e}+Q\right)^{2} f_{e^{+}} \\
\lambda_{n} \simeq & \frac{1}{2 \pi^{3}}|M|^{2} n_{n} \int_{m_{e}}^{Q} d E_{e} E_{e} p_{e}\left(Q-E_{e}\right)^{2} \\
& \times\left(1-f_{e}\right),
\end{aligned}
$$

where $Q=m_{n}-m_{p}$ is the mass difference between neutron and proton, $n_{i}=$ $2\left(m_{i} T / 2 \pi\right)^{3 / 2} \exp \left(\eta_{i}\right)$ is the number density of neutrons and protons in the nondegenerate limit, and $\eta_{i}=\left(\mu_{i}-m_{i}\right) / T$ is the reduced chemical potential. The above approximate rates are frequently cited in the literature to discuss the kinetic equilibrium for the $\beta$-processes and the emissivity of neutrino emission, even though its validity should be checked carefully [4, 5, 16].

At $m_{e}, \mu_{e}, Q<T$, the above reaction rates can be simplified further into

$$
\begin{aligned}
\lambda_{e^{-} p} \simeq & 2^{-1.5} \pi^{-4.5}|M|^{2} m_{p}^{1.5} T^{6.5} \\
& \times \exp \left(\eta_{p}+\mu_{e} / T-Q / T\right) \\
& \times[I(4)+2 I(3) Q / T], \\
\lambda_{e^{+} n} \simeq & 2^{-1.5} \pi^{-4.5}|M|^{2} m_{n}^{1.5} T^{6.5} \\
& \times \exp \left(\eta_{n}-\mu_{e} / T\right) \\
& \times[I(4)+2 I(3) Q / T], \\
\lambda_{n} \simeq & 1.63 \times 2^{-1.5} \pi^{-4.5}|M|^{2} m_{n}^{1.5} T^{1.5} m_{e}^{5},
\end{aligned}
$$

here $I(3)=7 \pi^{4} / 120$, and $I(4)=45 \zeta(5) / 2, \zeta(5)=1.037$ is the Riemann $\zeta$-function. The definition of $I(n)$ is $I(n) \equiv \int_{0}^{\infty}\left(e^{x}+1\right)^{-1} x^{n} d x$. This integral can be found in Gradshteyn and Ryzhik [24].

In the elastic limit, $n_{p}$ in Eq. (12) is replaced by

$$
\eta_{p n}=\left(n_{p}-n_{n}\right) /\left(1-e^{\left(\eta_{n}-\eta_{p}\right) / T}\right)
$$

It is generally believed that electron capture rate under the elastic approximation in some sense introduces the effects of the degeneracy of baryons, so it should be more accurate than that under the nondegenerate approximation. Suppose that the nuclei are dissolved 


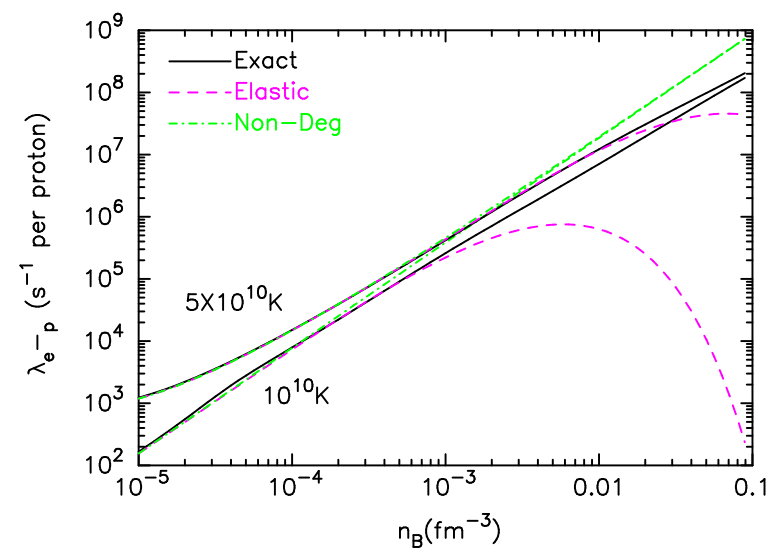

FIG. 2: Electron capture rates on protons in the dense subnuclear matter out of the chemical equilibrium as a function of the baryon number density $n_{\mathrm{B}}$ at different temperatures. The electron fraction $Y_{e}$ is taken to be 0.3. The solid curves show the results from Eq. (5), the dashed curves are the elastic results from Eq. (18), the dot-dashed curves are the nondegenerate results from Eq. (12).

completely into nucleons at high temperature. Figure 2 shows the differences between our electron capture rate in the dense subnuclear matter out of the chemical equilibrium and the previous approximate results. The electron fraction $Y_{e}=\left(n_{e^{-}}-n_{e^{+}}\right) / n_{\mathrm{B}}$ is assumed to be 0.3. The number density of particles at any degeneracy is expressed in terms of the Fermi-Dirac functions [25]. The multidimensional integrations and the Fermi-Dirac functions are calculated using the mixture of Gauss-Legendre and Gauss-Laguerre quadratures [25]. From Fig. 2, it is evident that there are great differences between the elastic results and the general results (Eq. 5) when baryons become degenerate. As shown in Eq. (18), the capture rates under the elastic approximation always decrease exponentially when nucleons become degenerate, which is qualitatively correct in the dense nuclear matter near the $\beta$-equilibrium. However, this conclusion is not correct obviously in the dense subnuclear matter out of the chemical equilibrium. The elastic approximation is also good when $\mu_{p} \simeq \mu_{n}$ because the favored energy transfer is zero. In short, the elastic approximation underestimates the electron capture rate, when baryons become degenerate. 


\section{CONDITIONS FOR KINETIC EQUILIBRIUM.}

\section{A. Chemical equilibrium condition for neutrino trapping.}

If neutrinos are trapped in a system, as what happens in the interior of a PNS, the inverse reactions corresponding to beta processes (11)-(3) by absorption of neutrinos can take place:

$$
\begin{aligned}
e^{-}+p & \rightleftharpoons n+\nu_{e}, \\
e^{+}+n & \rightleftharpoons p+\bar{\nu}_{e}, \\
n & \rightleftharpoons p+e^{-}+\bar{\nu}_{e}
\end{aligned}
$$

and their rates are equal to these of the corresponding beta reactions when the system reaches the chemical equilibrium. Because both the photons and neutrinos are trapped, the chemical equilibriums $\gamma+\gamma \rightleftharpoons e^{+}+e^{-} \rightleftharpoons \nu_{e}+\bar{\nu}_{e} \operatorname{give}\left(\mu_{\gamma}=0\right)$,

$$
\begin{aligned}
& \mu_{e^{+}}=-\mu_{e^{-}}, \\
& \mu_{\bar{\nu}_{e}}=-\mu_{\nu_{e}} .
\end{aligned}
$$

Based on the standard arguments in the theory of thermodynamics, the chemical equilibrium of any one of the above reversible reactions (19)-(21) gives the same chemical equilibrium condition for both cold $n p e^{-}$and hot $n p e^{ \pm}$gases,

$$
\mu_{n}+\mu_{\nu_{e}}=\mu_{p}+\mu_{e}
$$

If the chemical potential of the trapped neutrinos is zero, the above equation reduces to

$$
\mu_{n}=\mu_{p}+\mu_{e}
$$

and the corresponding number density of the trapped neutrinos is written as

$$
n_{\nu_{e}}=n_{\bar{\nu}_{e}}=\frac{T^{3}}{2 \pi^{2}} \int_{0}^{\infty} \frac{x^{2} d x}{e^{x}+1}=\frac{3 T^{3}}{4 \pi^{2}} \zeta(3),
$$

where $\zeta(3) \simeq 1.202$. It is clearly shown in Eq. (26) that the number density of the trapped neutrino is not zero at all when their chemical potential is zero. Only when $T \rightarrow 0, n_{\nu_{e}}=$ $n_{\bar{\nu}_{e}} \rightarrow 0$. Therefore, Eq. (25) can also be understood as the chemical equilibrium condition for cold $n p e^{-}$gas under $\beta$-equilibrium when neutrinos can escape freely from the system. 


\section{B. Kinetic equilibrium condition for neutrino escaping.}

In principle, if the matter is transparent to neutrinos which carry energy away, the equilibrium of the reactions (11)-(B) can not be treated as a chemical equilibrium problem [20]. Suppose that the dynamic time scale of the system under consideration is greater than that of the reactions (11)-(3), the general condition for the $\beta$-equilibrium is given by

$$
\begin{aligned}
& \lambda_{e^{-} p}\left(\mu_{n}, \mu_{p}, \mu_{e}, T\right) \\
& =\lambda_{e^{+} n}\left(\mu_{n}, \mu_{p}, \mu_{e}, T\right)+\lambda_{n}\left(\mu_{n}, \mu_{p}, \mu_{e}, T\right) .
\end{aligned}
$$

Given $n_{\mathrm{B}}$ and $T$, there are two additional trivial conditions. One is the conservation of the baryon number,

$$
n_{n}\left(\mu_{n}, T\right)+n_{p}\left(\mu_{p}, T\right)=n_{\mathrm{B}},
$$

the other is the charge neutrality,

$$
n_{e^{-}}\left(\mu_{e}, T\right)-n_{e^{+}}\left(\mu_{e}, T\right)=n_{p}\left(\mu_{p}, T\right)
$$

Consequently, the chemical potentials of neutron, proton and electron $\left(\mu_{n}, \mu_{p}, \mu_{e}\right)$ can be determined by a set of closed equations, i.e. , Eqs. (27)-(29).

\section{ANALYTIC $\beta$-EQUILIBRIUM CONDITIONS FOR NEUTRINO ESCAPING.}

\section{A. $\beta$-equilibrium condition for cold $n p e^{-}$gas.}

For ideal, cold $n p e^{-}$gas, we can set the chemical potential of the neutrinos in Eq. (24) to zero because their number density is zero at $T=0$ (see Eq. (26) ), therefore, the chemical equilibrium conditions for the ideal, cold npe $e^{-}$gas is Eq. (25), i.e. , $\mu_{n}=\mu_{p}+\mu_{e}$ [20].

In the following, we will re-derive the chemical equilibrium condition for the cold $n p e^{-}$ gas Eq. (25) from the viewpoint of the equality of the reaction rates of the beta processes. In the interior of an old neutron star, the electrons are degenerate $\left(E_{e} \gg T\right)$, thus the number density of positrons are exponentially depressed. As the typical energy of emitted neutrinos is of order the temperature, the energy and the momentum of the emitted neutrinos can be neglected comparing to the Fermi energy of particles, i.e. , $E_{p}+E_{e}=E_{n}$, and $\overrightarrow{p_{p}}+\overrightarrow{p_{e}}=\overrightarrow{p_{n}}$. So the kinetic equilibrium requires $\lambda_{e^{-} p}=\lambda_{n}$, therefore,

$$
0=\lambda_{e^{-} p}-\lambda_{n}
$$




$$
\begin{aligned}
\simeq & 2 \int \prod_{i=1}^{4}\left[\frac{d^{3} \vec{p}_{i}}{(2 \pi)^{3}}\right](2 \pi)^{4}|M|^{2} \delta^{(4)}\left(P_{e}+P_{p}-P_{n}\right) \\
& {\left[f_{p}\left(1-f_{n}\right) f_{e}-\left(1-f_{p}\right) f_{n}\left(1-f_{e}\right)\right] } \\
\simeq & 2 \int \prod_{i=1}^{4}\left[\frac{d^{3} \vec{p}_{i}}{(2 \pi)^{3}}\right](2 \pi)^{4}|M|^{2} \delta^{(4)}\left(P_{e}+P_{p}-P_{n}\right) \\
& f_{p}\left(1-f_{n}\right)\left(1-f_{e}\right) e^{-E_{e}}\left[e^{\mu_{e} / T}-e^{\left(\mu_{n}-\mu_{p}\right) / T}\right] .
\end{aligned}
$$

In the above derivations, we use the even property of the Dirac-delta function, i.e. , $\delta^{(4)}\left(P_{e}+\right.$ $\left.P_{p}-P_{n}\right)=\delta^{(4)}\left(P_{n}-P_{e}-P_{p}\right)$. It is evident that Eq. (30) eventually results in the well-known chemical equilibrium condition Eq. (25) which is generally used to determine the equation of state of the dense matter in the interior of an old neutron star.

\section{B. Analytic steady state equilibrium condition for hot $n p e^{ \pm}$gas.}

It is well known that in a system with plenty of positrons, the $e^{ \pm}$pair must not be degenerate $\left(T>E_{e}\right)$, if not, the number density of positrons will decrease exponentially, therefore, $\exp \left(\left(E_{e}-\mu_{e}\right) / T\right) \geq 1$. Before we derive the analytic dynamical equilibrium condition for such system from which the emitted neutrinos can escape freely, we first explain why the condition is not the well-known result of Eq. (25). Suppose that Eq. (25) is satisfied, for the reason described in §III.A., the chemical potential of the trapped neutrinos is zero, thus the ratio of the number density of the trapped neutrinos to that of the electrons is about $T^{3} / E_{e}^{3}>1$, if the electrons are not degenerate! This conclusion is obviously contradictory to the precondition that the system is transparent to neutrinos.

In such system, comparing to the rate of positron capture by neutrons, the rate of neutron decay could be neglected before reaching the degenerate limit. Thus the $\beta$-equilibrium condition for hot npe $e^{ \pm}$gas is [4, 5]

$$
\lambda_{e^{-} p}=\lambda_{e^{+} n}
$$

if the hot electron-positron plasma with nucleon is transparent to neutrinos. Another good approximation is the elastic approximation, that is, the energy of the emitted neutrinos is of order that of the captured $e^{ \pm}$, i.e. , $E_{\nu} \simeq E_{e}, E_{\bar{\nu}} \simeq E_{e^{+}}$, and thus $E_{n} \simeq E_{p}$.

Under these approximations, the kinetic equilibrium condition Eq. (31) gives

$$
0=\lambda_{e^{-} p}-\lambda_{e^{+} n}
$$




$$
\begin{aligned}
\simeq & 2 \int \prod_{i=1}^{4}\left[\frac{d^{3} \overrightarrow{p_{i}}}{(2 \pi)^{3}}\right](2 \pi)^{4}|M|^{2} \delta^{(4)}\left(P_{e}+P_{p}-P_{n}-P_{\nu_{e}}\right) \\
& {\left[f_{p} f_{e}\left(1-f_{n}\right)-\left(1-f_{p}\right) f_{e^{+}} f_{n}\right] } \\
\simeq & 2 \int \prod_{i=1}^{4}\left[\frac{d^{3} \overrightarrow{p_{i}}}{(2 \pi)^{3}}\right](2 \pi)^{4}|M|^{2} \delta^{(4)}\left(P_{e}+P_{p}-P_{n}-P_{\nu_{e}}\right) \\
& f_{p}\left(1-f_{n}\right) f_{e^{+}} \\
& {\left[\frac{e^{\left(E_{e}+\mu_{e}\right) / T}+1}{e^{\left(E_{e}-\mu_{e}\right) / T+1}}-e^{\left(\left(E_{p}-E_{n}\right)-\left(\mu_{p}-\mu_{n}\right)\right) / T}\right] } \\
\simeq & 2 \int \prod_{i=1}^{4}\left[\frac{d^{3} \vec{p}_{i}}{(2 \pi)^{3}}\right](2 \pi)^{4}|M|^{2} \delta^{(4)}\left(P_{e}+P_{p}-P_{n}-P_{\nu_{e}}\right) \\
& f_{p}\left(1-f_{n}\right) f_{e^{+}}\left[e^{2 \mu_{e} / T}-e^{\left(\mu_{n}-\mu_{p}\right) / T}\right] .
\end{aligned}
$$

Therefore, from Eq. (32) we obtain the beta-equilibrium condition for the $e^{ \pm}$plasma with nucleons

$$
\mu_{n}=\mu_{p}+2 \mu_{e}
$$

It should be emphasized that during the above derivation, it is not assumed whether the baryons are degenerate or not. On the other hand, Eq. (333) is still valid under the degeneracy of baryons, which is neglected completely in the approximate reaction rates at the beginning. However, it should be pointed out that wherever baryons are degenerate in astrophysics, neutrinos are generally trapped and likely thermalized. In these post-neutrino trapping environments, the analytic steady state equilibrium condition we derived here is not valid, because the effects of neutrino trapping are not included in our consideration. It is not a surprise to notice that the analytic equilibrium condition Eq. (33) can also be drawn in the completely nondegenerate limit. Setting the equality of Eq. (15) and Eq. (16), the equilibrium condition is also obtained. In the following, we have another heuristic but not very strict derivation. If all particles are nondegenerate, we have [20, 21]

$$
\begin{aligned}
& \lambda_{e^{-} p} \propto n_{e^{-}} n_{p} \propto \exp \left(\eta_{p}+\eta_{e}\right), \\
& \lambda_{e^{+} n} \propto n_{e^{+}} n_{n} \propto \exp \left(\eta_{n}-\eta_{e}\right) .
\end{aligned}
$$

Setting the equality of Eq. (34) and Eq. (35), the analytic condition Eq. (33) is obtained again.

The advantage of having the analytic equilibrium condition at hand is obvious. For instance, we can derive some useful formula in the nondegenerate limit of baryons. In such 
limit, using the Saha equation gives the ratio of neutron baryon number density to that of proton (e.g. [26]),

$$
\frac{n_{n}}{n_{p}} \equiv 1-\frac{1}{Y_{e}}=e^{\frac{\mu_{n}-\mu_{p}-Q}{T}}=e^{\frac{2 \mu_{e}-Q}{T}} .
$$

At the last step, the analytic equilibrium condition Eq. (33) is applied. On the other hand, for the relativistic $e^{ \pm}$, there exits an exact expression for the electric charge density in terms of the chemical potential of electrons (e.g. [27]),

$$
n_{e^{-}}-n_{e^{+}} \equiv n_{\mathrm{B}} Y_{e}=\frac{T^{3}}{3 \pi^{2}}\left[\left(\frac{\mu_{e}}{T}\right)^{3}+\pi^{2}\left(\frac{\mu_{e}}{T}\right)\right]
$$

Substituting Eq. (36) into Eq. (37), we have only one simple equation to determine the electron chemical potential $\mu_{e}$,

$$
\frac{T^{3}}{3 \pi^{2}}\left[\left(\frac{\mu_{e}}{T}\right)^{3}+\pi^{2}\left(\frac{\mu_{e}}{T}\right)\right]-\frac{n_{\mathrm{B}}}{1+e^{\left(2 \mu_{e}-Q\right) / T}}=0 .
$$

After that, the electron fraction $Y_{e}$ can be directly calculated from Eq. (37).

At $\mu_{e} / T<1$ and $\left(2 \mu_{e}-Q\right) / T<1$, Eq. (38) can be simplified further,

$$
Y_{e}=\frac{1}{2} \frac{(1+0.5 Q / T)}{\left(1+1.5 n_{\mathrm{B}} / T^{3}\right)} .
$$

Equating the rates of $e^{-}$and $e^{+}$captures in the region of mild degeneracy, Beloborodov [5] obtained a similar result to Eq. (399) ( see Eq. (11) in his paper). In a word, using the well known results and our new result Eq. (33), we re-derive a similar previous result in a different way. From this consistence, it turns out that Eq. (33) is correct and the approximate formula Eq. (38) is more accurate than the previous result.

In the following, we check the validity of Eq. (33) by numerical calculations. As before, suppose that the nuclei are dissolved completely into nucleons at high temperature. Given $n_{\mathrm{B}}$ and $T$, the electron fraction $Y_{e}$ can be determined by two different sets of the equilibrium conditions. One set consists of Eqs. (27)-(29). In Eq. (27), the $\beta$ reaction rates are calculated based on the reaction rates Eqs. (15)-(17), or the approximate rates Eqs. (12)-(14), respectively. The other set consists of our analytic equation Eq. (33), and Eqs. (28)-(29). Figure 3 shows $Y_{e}$ and the reduced chemical potentials $\eta_{i}(i=n, p, e)$ versus the baryon number density at different temperatures. In the upper panel of Fig. (31), the solid lines show the results from Eqs. (27)-(29) and Eqs. (5)-(17) for the $\beta$ reaction rates. The dot-dashed curves are from Eqs. (27)-(29), but the approximate reaction rates in the nondegenerate limit Eqs. (12)-(14) are used. The dotted lines represent the results from Eq. (33) and Eqs. (28)-(29). It is 


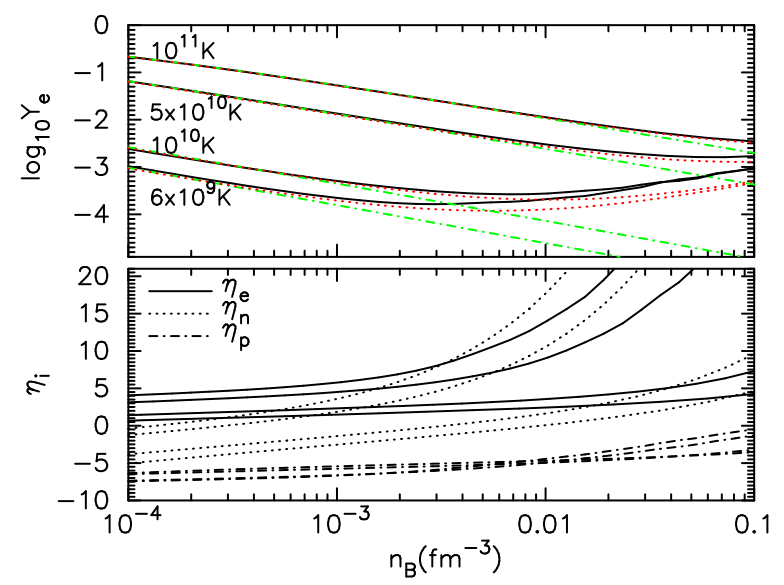

FIG. 3: Upper panel: Electron fraction $Y_{e}$ under $\beta$-equilibrium versus the baryon number density $n_{\mathrm{B}}$ at different temperatures. The solid curves show the results from Eqs. (15)-(77), the dotted curves are from the analytic $\beta$-equilibrium condition Eq. (33), and the dot-dashed curves are from the nondegenerate approximation of baryons. Lower panel: The reduced chemical potentials of electrons, neutrons, and protons $\eta_{i}$ as a function of the baryon number density at different temperatures. From top to bottom, the lines correspond to the results of $T=6 \times 10^{9}, 10^{10}$, $5 \times 10^{10}, 10^{11} \mathrm{~K}$.

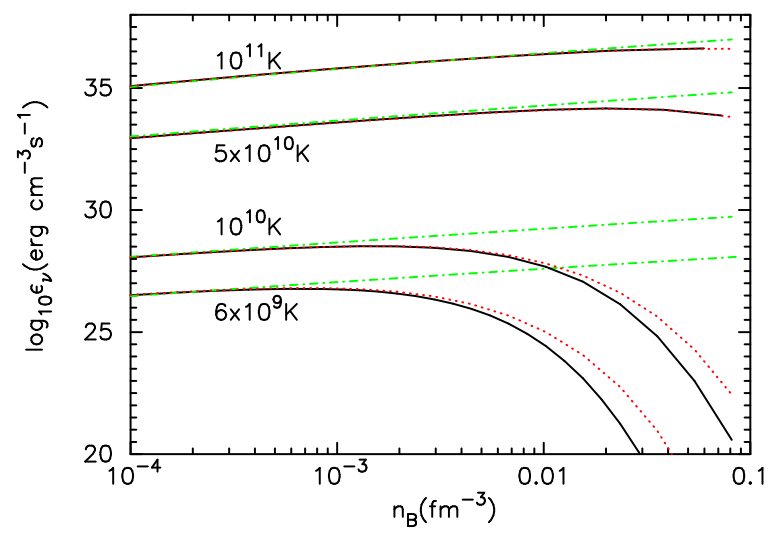

FIG. 4: The neutrino emissivity $\epsilon_{\nu}$ versus the baryon number density at different temperatures. The lines are the same as those of the upper panel in Fig. 3

evident that the results from the analytic condition are almost consistent with the general numerical results. As expected, the difference occurs only in the regime where electrons become degenerate, beyond which, the condition for the analytic formula Eq. (33) is not satisfied any longer and the contribution from the neutron decay can not be neglected. As shown in the lower panel of Fig. 3, neutrons become degenerate as electrons do. The 
particles become degenerate if their reduced chemical potential exceeds the temperature. For electrons and nucleons, their degenerate number densities are estimated to be

$$
\begin{aligned}
n_{e}^{\mathrm{deg}} & =p_{e}^{3} / 3 \pi^{2} \simeq T^{3} / 3 \pi^{2} \\
& \simeq 9.0 \times 10^{-6}\left(\frac{T}{10^{11} \mathrm{~K}}\right)^{3} \mathrm{fm}^{-3}, \\
n_{n, p}^{\mathrm{deg}} & =p_{n, p}^{3} / 3 \pi^{2} \simeq\left(2 m_{n, p} T\right)^{3 / 2} / 3 \pi^{2} \\
& \simeq 2.8 \times 10^{-3}\left(\frac{T}{10^{11} \mathrm{~K}}\right)^{3 / 2} \mathrm{fm}^{-3} .
\end{aligned}
$$

In the same regime, there are great differences (of several orders) between our results and the previous results in which the degeneracy of nucleons is completely neglected. In any case, Fig. [3 evidently shows that the results from the analytic condition are much more accurate than those from the approximate rates in all the parameter regions.

The total neutrino emissivity under $\beta$-equilibrium is shown in Fig. [. Compared with the general numerical results, both approximate methods overestimate the rate of the neutrino emission because neglect of the degeneracy of particles increases the phase space for the

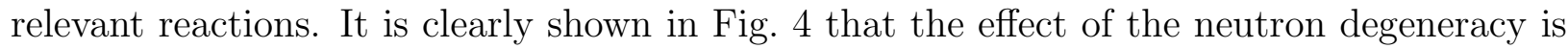
much more important than that of electrons, if such conditions are satisfied.

\section{CONCLUSIONS AND DISCUSSIONS}

In this work, using the structure function formalism developed by Reddy et al. [22], we derive the rates of the $\beta$ processes which including $e^{ \pm}$captures and neutron decay in the dense subnuclear matter for all participating particles are at arbitrary degeneracy. For this purpose, the difference between the mass of neutron and proton is kept in the structure function (see Eq. (11)), which is neglected originally in Reddy et al. 22]. Comparing to our reaction rates, the previous approximations have been checked. Especially, the electron capture rate under the elastic approximation which is put forward to include the effects of the degeneracy of baryons differs dramatically from our results when baryons become degenerate and before the dense matter reaches the $\beta$-equilibrium. Generally speaking, the elastic approximation underestimates the electron capture rate when baryons become degenerate.

Most of interest, we find an analytic steady state equilibrium condition for $e^{ \pm}$plasma with nucleons, if the system is transparent to the emitted neutrinos. This result is valid 
when electrons are nondegenerate or mildly degenerate, but it has nothing to do with the degeneracy of baryons. Basically, it is a good result for $e^{ \pm}$plasma with nucleons, because when electron becomes degenerate, the number density of positron decreases exponentially, then the system can not be called as $e^{ \pm}$plasma. If the nucleons are nondegenerate, we further obtain only one simple equation for determining the electron chemical potential and electron fraction, which shows the great advantage of our analytic equilibrium condition.

So far, there are four analytic beta equilibrium or steady state equilibrium conditions for $n p e^{ \pm}$gas in different astrophysical circumstances. They are summarized as follows: (1), If the neutrinos are completely trapped in a system, such as in the interior of a PNS, $\mu_{n}+\mu_{\nu_{e}}=\mu_{p}+\mu_{e}$. (2), In the cold npe- gas under beta equilibrium, $\mu_{n}=\mu_{p}+\mu_{e}$. (3), If the neutrinos are partially trapped, and the chemical potential of the trapped neutrinos is zero $\left(\mu_{\nu_{e}}=0\right), \mu_{n}=\mu_{p}+\mu_{e}$. (4), If the neutrinos can escape freely from the system with plenty of $\mathrm{e}^{ \pm}$pairs, $\mu_{n}=\mu_{p}+2 \mu_{e}$, which is the main result of this work.

\section{Acknowledgments}

The author would like to thank the anonymous referee for her/his constructive suggestions which are very helpful to improve this manuscript, and Dr. Ramesh Narayan, Dr. Jeremy Heyl and Dr. Rosalba Perna for many discussions, Dr. Dong Lai for comments, and Dr. David Rusin for a critical reading of this manuscript. The author acknowledges the hospitality of Harvard-Smithsonian Center for Astrophysics. This work is partially supported by the Special Funds for Major State Research Projects, and the National Natural Science Foundation (10233030).

[1] P. Mészáros, Annu. Rev. Astron. Astrophys. 40, 137 (2002).

[2] H. Janka, K. Kifonidis, and M. Rampp, in Physics of Neutron Star Interiors (Springer-Verlag, Heidelberg,2001), pp. 363, A. Burrows, Nature (London) 403, 727 (2000).

[3] R. Narayan, B. Paczynski, and T. Piran, Astrophys. J. Lett. 395, L83 (1992); R. Popham, S. E. Woosley, and C. Fryer, Astrophys. J. 518, 356 (1999); R. Narayan, T. Piran, and P. Kumar, Astrophys. J. 557, 949 (2001); K. Kohri and S. Mineshige, Astrophys. J. 577, 311 (2002); T. Di Matteo, R. Perna, and R. Narayan, Astrophys. J. 579, 706 (2002); S. Setiawan, 
M. Ruffert, and H.-T. Janka, Mon. Not. Roy. Astron. Soc. 352, 753 (2004), W. H. Lee, E. Ramirez-Ruiz, and D. Page, Astrophys. J. Lett. 608, L5 (2004).

[4] J. Pruet, S. E. Woosley, and R. D. Hoffman, Astrophys. J. 586, 1254 (2003),

[5] A. M. Beloborodov, Astrophys. J. 588, 931 (2003),

[6] S. Fujimoto, M. Hashimoto, K. Arai, and R. Matsuba, Astrophys. J. 614, 847 (2004); R. Matsuba, K. F. S. Arai, and M. Hashimoto, Pub. Astron. Soc. Japan 56, 407 (2004); R. Surman and G. C. McLaughlin, Astrophys. J. 603, 611 (2004); S. Inoue, N. Iwamoto, M. Orito, and M. Terasawa, Astrophys. J. 595, 294 (2003).

[7] E. V. Derishev, V. V. Kocharovsky, and V. V. Kocharovsky, Astrophys. J. 521, 640 (1999).

[8] J. Pruet and N. Dalal, Astrophys. J. 573, 770 (2002).

[9] G. M. Fuller, J. Pruet, and K. Abazajian, Phys. Rev. Lett. 85, 2673 (2000).

[10] J. N. Bahcall and P. Mészáros, Phys. Rev. Lett. 85, 1362 (2000).

[11] B. L. Friman and O. V. Maxwell, Astrophys. J. 232, 541 (1979); J. M. Lattimer, C. J. Pethick, M. Prakash, and P. Haensel, Phys. Rev. Lett. 66, 2701 (1991); D. G. Yakovlev, A. D. Kaminker, O. Y. Gnedin, and P. Haensel, Phys. Rep. 354, 1 (2001).

[12] A. M. Beloborodov, Astrophys. J. Lett. 585, L19 (2003).

[13] K. Langanke and G. Martínez-Pinedo, Rev. Mod. Phys. 75, 819 (2003).

[14] S. E. Woosley, A. Heger, and T. A. Weaver, Rev. Mod. Phys. 74, 1015 (2002); M. Prakash et al. , Phys. Rep. 280, 1 (1997); S. I. Dutta, S. Ratković, and M. Prakash, Phys. Rev. D 69, 023005 (2004); S. Ratković, S. Iyer Dutta, and M. Prakash, Phys. Rev. D 67, 123002 (2003); C. L. Fryer and P. Mészáros, Astrophys. J. Lett. 588, L25 (2003); Y.-Z. Qian and S. E. Woosley, Astrophys. J. 471, 331 (1996).

[15] V. Pinaev, Soviet Physics JETP 18, 377 (1964); C. J. Hansen, Astrophys. Space Sci. 1, 499 (1968).

[16] V. S. Imshennik, D. K. Nadezhin, and V. S. Pinaev, Soviet Astron. 10, 970 (1967).

[17] R. F. Sawyer, Phys. Rev. D 68, 063001 (2003).

[18] R. G. Couch and G. L. Loumos, Astrophys. J. 194, 385 (1974); K. Takahashi, M. F. El Eid, and W. Hillebrandt, Astron. Astrophys. 67, 185 (1978); G. M. Fuller, W. A. Fowler, and M. J. Newman, Astrophys. J. Suppl. Ser. 42, 447 (1980); J. Gutierrez, E. Garcia-Berro, I. J. Iben et al. , Astrophys. J. 459, 701 (1996); Z. Q. Luo and Q. H. Peng, Sci. Sinica A 39, 776 (1996); E. Bravo and D. García-Senz, Mon. Not. Roy. Astron. Soc. 307, 984 (1999); N. Itoh, 
N. Tomizawa, M. Tamamura et al. , Astrophys. J. 579, 380 (2002).

[19] C. J. Horowitz and K. Wehrberger, Nucl. Phys. A531, 665 (1991); C. J. Horowitz and K. Wehrberger, Phys. Rev. Lett. 66, 272 (1991); C. J. Horowitz and J. Piekarewicz, Phys. Lett. B 226, 236 (1992); C. J. Horowitz and J. Piekarewicz, Nuclear Physics A 536, 669 (1992).

[20] S. L. Shapiro and S. A. Teukolsky, Black holes, white dwarfs, and neutron stars: The physics of compact objects (Wiley-Interscience, New York, 1983).

[21] S. W. Bruenn, Astrophys. J. Suppl. Ser. 58, 771 (1985).

[22] S. Reddy, M. Prakash, and J. M. Lattimer, Phys. Rev. D 58, 13009 (1998).

[23] A. Burrows and R. F. Sawyer, Phys. Rev. C 58, 554 (1998).

[24] I. S. Gradshteyn and I. M. Ryzhik, Table of integrals, series and products (Academic Press, New York, 1965).

[25] J. M. Aparicio, Astrophys. J. Suppl. Ser. 117, 627 (1998).

[26] G. M. Fuller, Astrophys. J. 252, 741 (1982).

[27] S. I. Blinnikov, N. V. Dunina-Barkovskaya, and D. K. Nadyozhin, Astrophys. J. Suppl. Ser. 106, 171 (1996). 